\title{
Stress inhomogeneity effect on fluid-induced fracture behaviour into weakly consolidated granular systems.
}

\author{
Paula A. Gago, ${ }^{1, *}$ Charalampos Konstantinou, ${ }^{2}, \dagger$ Giovanna Biscontin, ${ }^{2}$ and Peter King ${ }^{1}$ \\ ${ }^{1}$ Department of Earth Science and Engineering, Imperial College, London, SWr 2BP, UK. \\ ${ }^{2}$ Department of Engineering, University of Cambridge, CB2 1PZ, UK.
}

\begin{abstract}
This paper studies the effect of stress inhomogeneity on the behaviour of fluid-driven fracture development in weakly consolidated granular systems. Using numerical models we investigate the change in fracture growth rate and fracture pattern structure in unconsolidated granular packs (also refered to as soft-sands) as a function of the change in the confining stresses applied to the system. Soft-sands do not usually behave like brittle, linear elastic materials and as a consequence, poro-elastic models are often not applicable to describe their behaviour. By making a distinction between "cohesive" and "compressive" grain-grain contact forces depending on their magnitude, we propose an expression that describes the fluid opening pressure as a function of the mean value and the standard deviation of the "compressive stress" distribution. We also show that the standard deviation of this distribution can be related with the extent to which fracture "branches" reach into the material.
\end{abstract}

Keywords: Soft Sand, Fracturing, Flow channels, Resolved CFD-DEM, Yielding Transition

Soft or weakly-consolidated sand refers to porous materials composed by particles (or grains) weakly held together to form a solid but that can be easily broken when subjected to stress. Some of the mechanisms contributing to hold particles together are grain-to-grain weak cementation (acting as a "glue") and the physical interlocking between particles that arises as a consequence of grains irregular shape. The understanding of the hydraulic fracture behaviour in such systems generates interest not only academically, but in many fields of engineering. Groundwater remediation, hydrocarbon extraction and in-situ leaching from mining, for example, deal with "soft-sand" formations and hence benefit from an ability to predict the material's response to fluid injection.

Various regimes have been reported [1-3] to describe the behaviour of granular packs (soft-sands) when a fluid is injected. While for low fluid injection rates these systems tend to behave as a conventional solid porous material with Darcy-like flow taking place, for higher inlet fluid pressures (which lead to higher flow rates) grains start rearranging, creating voids in the system. These voids in turn re-arrange creating complex branched-like structures or "fractures". These fractures have been described $[1,3]$ as taking different geometries. Generally grain displacement starts by forming patterns with a circular symmetry around the fluid inlet. As fluid pressure increases instabilities arise leading to the formation of "fingers" or branched-like patterns. This behaviour has been observed both, numerically [3], simulating air injection into a not cohesive granular pack, and experimentally [1], where a mixture of water and glycerin was injected into dried granular media in a quasi twodimensional Hele-Shaw cell.

\footnotetext{
* paulaalejandrayo@gmail.com

$\dagger$ ck494@cam.ac.uk
}

It is known that granular systems do not behave as conventional brittle, linear elastic materials. Their behaviour is heavily controlled by dissipative (frictional) forces and a highly inhomogeneous stress distribution [46]. Due to these intrinsic characteristics, poro-elastic models do not usually describe well the fracture behaviour of these systems under stress.

In this paper we demonstrate how the distribution of stress acting on the systems relates to both, the critical opening pressure and the fracture behaviour. To this end, we numerically simulate the process of fluid injection into a quasi two dimensional Hele-Shaw cell filled with weakly cemented granular matter. The system stress is modified by applying external forces through piston-like walls (Fig.1). We measure the stress distribution of the grains under different stress conditions and characterise the mean value and standard deviation of this distribution (Fig.4). We then measure the fracture area as a function of the fluid inlet pressure and show that this can be scaled using the mean value and the standard deviation of the stress distribution (Fig. 3). We also show how different stress conditions lead to different fracture patterns (Fig. 2) and calculate the radius of gyration of the obtained patterns obtained (Fig. 5).

To create the initial pack we first create a loose configuration $\left(N=10^{4}\right)$ of spherical grains (with diameters following a truncated gaussian distribution in the range of $0.9-1.8 \mathrm{~mm}$ with mean diameter $d=1.363 \mathrm{~mm}$ and a standard deviation $\sigma_{d} \sim 0.3 \mathrm{~mm}$ ) placed between two parallel plates separated by a constant distance of approximately 2 mean grain diameters. The pack is compressed in the plane using 8 piston-like octagonal walls (Fig. 1 (a)). These walls apply a constant lateral force $\left(F_{l}\right)$ to the system. The central inlet, with a radius $r_{i}=3.5 \mathrm{~mm}$, is created by preventing particles from the pack going into this region (using a wall-like potential) while compaction takes place. Once at equilibrium, the lateral walls are fixed and a constant vertical force $\left(F_{v}\right)$ is applied through 


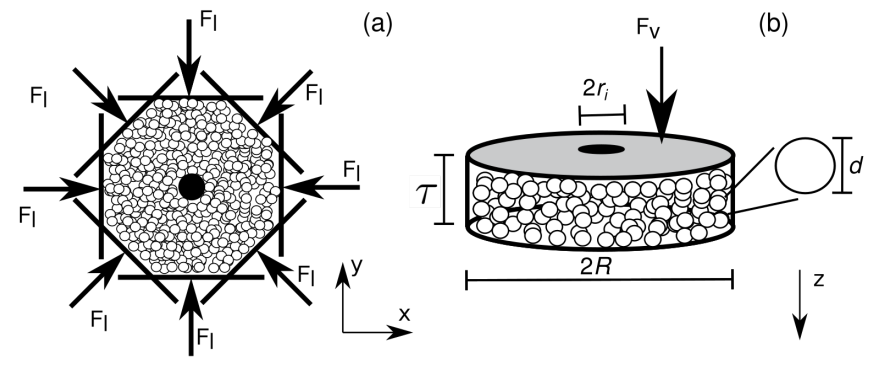

FIG. 1. Schematic representation of the system setup. (a) An initially loose pack of particles confined between two parallel plates separated a constant distance is compacted using eight piston-like walls applying a constant lateral force $\left(F_{l}\right)$ until a static configuration is reached. Lateral walls are fixed at this point. (b) A constant vertical force $\left(F_{v}\right)$ is applied to the upper wall of the cell to obtain a new equilibrium configuration. All walls in the system become fixed at this point. Grain mean diameter $d=1.363 \mathrm{~mm}, R \approx 4.4 \mathrm{~cm}, \tau \approx 2.4 \mathrm{~mm}$ $\ll R(\tau \approx 2 d), r_{i}=3.5 \mathrm{~mm}$.

the top wall (Fig. 1 (b)). All walls are fixed once a new equilibrium state is reached. The cell obtained has a radius $R \approx 4.4 \mathrm{~cm}$ and a thickness $\tau \approx 2.4 \mathrm{~mm} \ll R$. Once the initial condition is reached fluid injection is started. A pressure $p_{i}$ is set in the inlet and a pressure $p_{0}=0$ on the external perimeter of the cell, which is open to fluid flow (but impermeable to grains). The top and bottom walls are considered impermeable (to grains and fluid). Different initial conditions are created by controlling the lateral $\left(F_{l}\right)$ and the vertical $\left(F_{v}\right)$ applied forces.

The numerical simulations are performed using the model presented in reference [7], to simulate the interaction of fluid and dense granular packs. The model is based on $C F D E M$ [8] open source software and couples LIGGGHTS $[9,10]$, a discrete element method (DEM) software that implement a molecular dynamics of soft spheres model [11], with openFoam [12], a finite element method of computational fluid dynamics $(C F D)$.

An incompressible, newtonian fluid, with a viscosity $\eta=0.1 \mathrm{cp}$ and a density $\rho=1000 \mathrm{k} \mathrm{m}^{-3}$ is simulated. It is worth to note here that, due to the way the method uses to calculate the fluid forces acting on the grains, this model requires a $C F D$ mesh refinement of at least four grid-blocks per particle diameter $[7,10]$. In this work a mesh refinement of $1 / 6$ of the mean particle diameter is used.

Within LIGGGHTS we use a Hertz model [11], for the frictional force between two grains in contact, and a SJKR (simplified Johnson-Kendall-Roberts) model $[13,14]$ to add weak inter-grain cementation. The addition this attractive force aims to resembling weakcementation, and is necessary to incorporate soft-sand characteristics into the system as, given the spherical shape of the grains there is not interlock or any other collective behaviour occurring otherwise. In the follow, and for simplicity, we will refer to this force as cohesion.

The addition of this cohesive contribution allows the
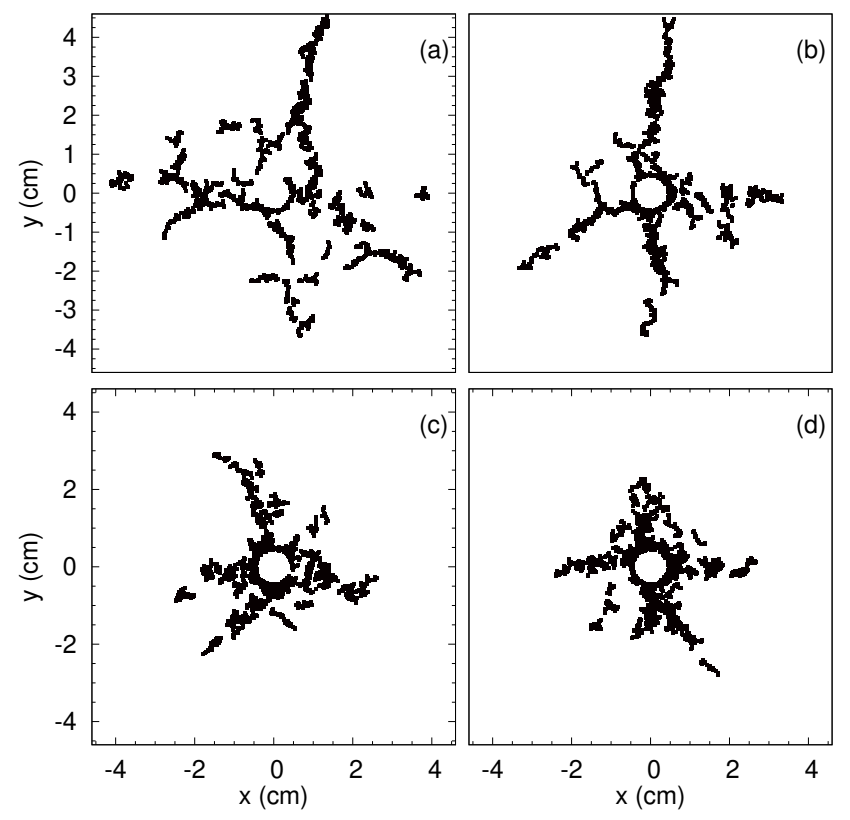

FIG. 2. Fracture patterns obtained for cells under different lateral forces $F_{l}=$ (a) $2 \mathrm{~N}$, (b) $4 \mathrm{~N}$, (c) $8 \mathrm{~N}$, (d) $10 \mathrm{~N}$. All patterns correspond to similar fracture area $\left(\approx 3 \mathrm{~cm}^{2}\right)$.

normal contact forces to take both negative and positive values.

As particles have zero velocity once the pack is created, the normal forces for two particles $i j$ in contact can be written as:

$$
f_{n}^{i j}=-\frac{4}{3}\left(\frac{2\left(1-\nu^{2}\right)^{2}}{Y}\right)^{-1}\left(\frac{1}{r_{i}}-\frac{1}{r_{j}}\right)^{-1 / 2} \delta^{3 / 2}+C_{h} A_{i j}
$$

where $\delta$ is the linear overlap between the two particles in contact, $Y\left(=5 \times 10^{6} \mathrm{Nm}^{-2}\right)$ is the Young's modulus, $\nu(=0.15)$ is the Poisson's ratio, $C_{h}\left(=3 \times 10^{5} \mathrm{~J} / \mathrm{m}^{3}\right)$ is the cohesion energy density and $A=\left(-\pi\left(\delta-r_{i}-r_{j}\right)(\delta+\right.$ $\left.\left.r_{i}-r_{j}\right)\left(\delta-r_{i}+r_{j}\right)\left(\delta+r_{i}+r_{j}\right) /\left(4 \delta^{2}\right)\right)$ is the contact area between the particles. The granular model also uses a restitution coefficient $\epsilon=0.7$ and a friction coefficient $\mu=0.7$.

Different stress initial conditions were created using four different lateral forces $\left(F_{l}\right)$ and five different vertical forces $\left(F_{v}\right)$. In the range of $25-30$ different inlet pressure $p_{i}$ values were simulated for each lateral/vertical force combination studied and five independent realisations were performed for each condition. The total injection time used was $0.01 \mathrm{~s}$, which was enough to allow the system to reach a stationary state, that is when the fracture growth stops.

Fig. 2 shows fracture patterns of equivalent area, obtained for different values of the lateral force $\left(F_{l}\right)$ increasing from (a) to (d).

To obtain these patterns, the protocol explained in Ref. [15] was followed. Briefly, the particle positions were mapped onto a $(482,482,6)$ cubic grid (corresponding to the $C F D$ mesh) and each grid-block was set with 

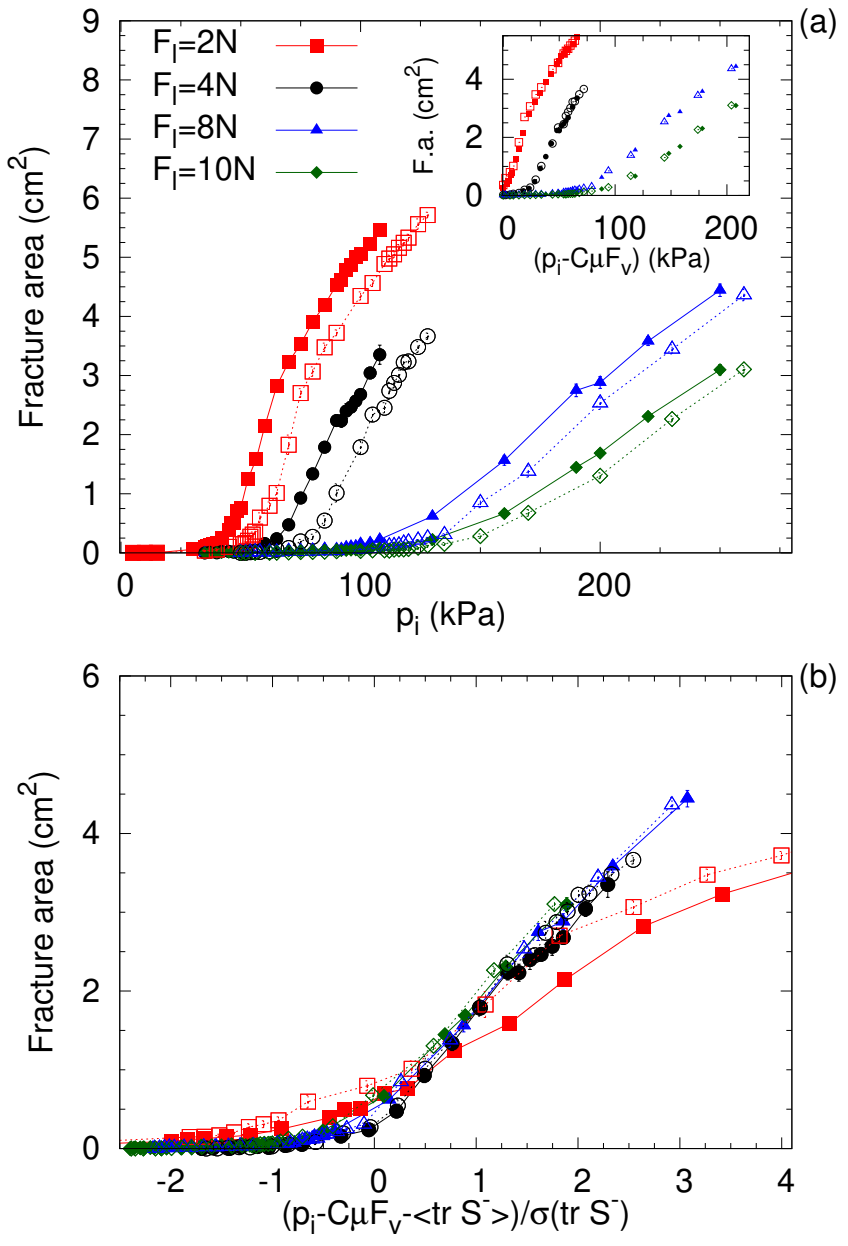

FIG. 3. (a) Fracture area as a function of inlet fluid pressure $p_{i}$ for initial conditions created with different lateral forces $F_{l}$ and two different vertical forces: $F_{v}=30 \mathrm{~N}$ (filled symbols) and $F_{v}=40 \mathrm{~N}$ (open symbols). The inset is as the main figure but as a function of $\left(p_{i}-C \mu F_{v}\right)$ (Eq. (2)), with $C=2$. (b) Fracture area as a function of $\left(p_{i}-p_{c}\right) / \sigma\left(\operatorname{tr}\left(S^{-}\right)\right)$, with $p_{c}$ defined in Eq. (4).

a value in the range $[0-1]$ proportional to the fraction of it that was filled by a particle. Clusters of adjacent grid-blocks with values smaller than 0.2 (i.e. containing mainly fluid) were localised. Fig. 2 depicts a longitudinal cut of this grid $(z=3)$ showing only those clusters formed for more than 70 grid-blocks (in this plane). The fracture area, defined as the area of the clusters in Fig. 2, as a function of the inlet pressure is shown in Fig. 3 (a). As expected, after reaching a critical injection pressure value $\left(p_{c}\right)$, particles start displacing and fractures are induced in the system.

An increment in the vertical confinement $\left(F_{v}\right)$ results in the fracture area, as a function of the inlet pressure, shifting towards higher values of pressure; this occurs without observable modifications on its growth "rate". In Ref. [15], the shift was attributed to be a consequence of the cell thickness being comparable with the mean diameter of particles, which inhibits or prevents the formation of force chains in the vertical direction. As a consequence of this, the vertical forces $\left(F_{v}\right)$ applied distribute homogeneously among the particles and frictional forces increase in proportion to normal forces. Given the frictional properties of the system, for particles to start displacing the fluid force acting on the grains needs to surpass the friction force. A change $\Delta F_{v}$ in the vertical force will result, therefore, in a change for the critical pressure given by:

$$
\Delta p_{c}\left(F_{v}\right)=C \mu \Delta F_{v}
$$

where $C$ is a constant that depends on the cell geometry and the particle size distribution, and $\mu$ is the system friction coefficient [15]. The inset on Fig. 3 (a) shows the same data as in the main figure but as a function of $p_{i}-C \mu F_{v}$, with $C=2$.

Although the narrowness of the cell prevents the formation of force chains in the vertical direction, in the plane direction force chains will develop and changes in the applied forces $\left(F_{l}\right)$ will distributed in an inhomogenous manner, stressing particles belonging to the chains more than those that do not. This causes a variation not only on the value of the critical pressure but also in the rate of growth of the fracture area for different $F_{l}$, as can be observed in the change of the slope of the curves in Fig. 3 (a).

Figure 4 (a) shows the distribution of normal forces $F_{n}$ acting on the particles' contacts for different values of the applied forces. For a purely non-cemented system $\left(C_{h}=0\right.$ in Eq. (1)), this distribution would only present negative values. Any positive values on this distribution are, therefore, due to the cohesive component of the contact force model. We refer to contacts with negative normal forces as "compressive" and contacts with positive normal contact forces as "cohesive". Open symbols in Fig. 4 (a) correspond to different values of the vertical force $F_{v}$, and show that the force distribution is barely affected by a change in $F_{v}$.

The stress moment tensor $S_{a b}^{i}$ of the particle $i$ is defined by:

$$
S_{a b}^{i}=-0.5 *\left(V^{i}\right)^{-1} \sum_{c} r_{a}^{i c} f_{b}^{i c}
$$

where the sum runs over all the particles $c$ in contact with the particle $i, V^{i}$ is the particle volume, $r_{a}^{i c}(a=\{x, y, z\})$ is the component in the $a$ direction of the vector connecting the center of the particle $i$ with the center of the particle $c$ and $f_{b}^{i c}$ is the $b$-th component of the contact force. As positive and negative forces correspond to different types of "contacts", we calculated two different stress tensors: $S^{-}$for the "compressive" forces and $S^{+}$ for the cohesive ones.

Figures 4 (b) and (c) show the distribution of the trace of $S^{-}$and $\left|S^{+}\right|$, respectively, for the different values of $F_{l}$ and $F_{v}$ applied. Insets in these figures show a suitable scaling for these distributions, using the mean value and the standard deviation $\sigma$ of the respective distributions. 

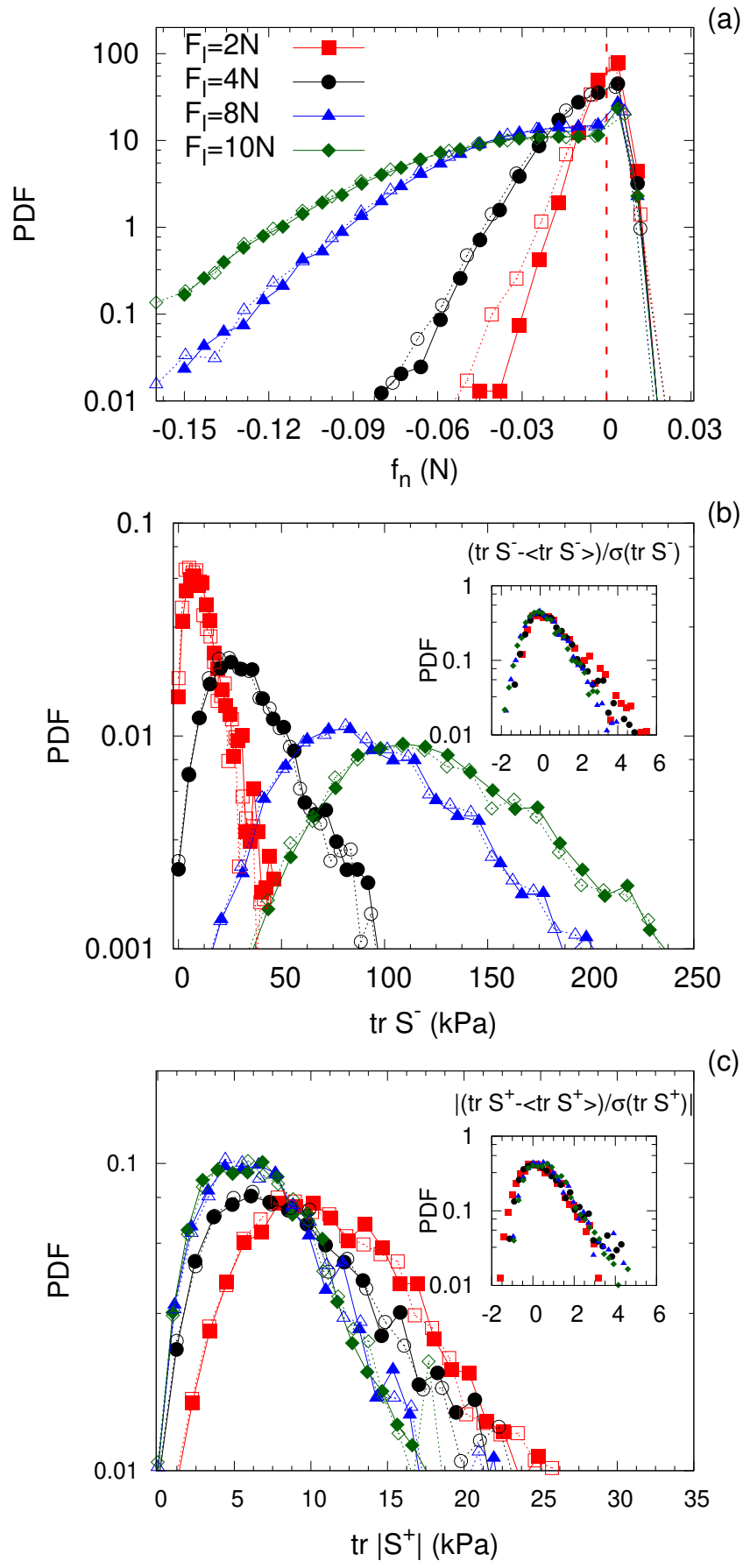

(c)

FIG. 4. (a) Distribution of the normal contact force $f_{n}$ (b) trace of the compressive stress $\operatorname{tr} S^{-}$and (c) trace of the cohesive stress $\operatorname{tr}\left|S^{+}\right|$, for initial conditions created using different lateral forces $\left(F_{l}\right)$ and two different vertical forces: $F_{v}=30 \mathrm{~N}$ (filled symbols) and $F_{v}=40 \mathrm{~N}$ (open symbols). Inset (b): distribution of $\left(\operatorname{tr} S^{-}-<\operatorname{tr} S^{-}>\right) / \sigma\left(\operatorname{tr} S^{-}\right)$. Inset (c) distribution of $\left(\operatorname{tr} S^{-}-<\operatorname{tr} S^{-}>\right) / \sigma\left(\operatorname{tr} S^{-}\right)$.
Figure 4 (b) shows that an increase in $F_{l}$ shifts the $S^{-}$ distribution towards higher values of stress and also increases the standard deviation $\sigma^{-}=\sigma\left(t r S^{-}\right)$in a similar proportion.

Figure 4 (c) shows that the cohesive stress $S^{+}$changes very little as a function of the applied forces, as it does its dispersion $\sigma^{+}=\sigma\left(\operatorname{tr} S^{+}\right)$. This suggests that this distribution may be a good candidate for defining the system strength. Further detailed work needs to be carried in order to establish and quantify this relation. Such an analysis is beyond the scope of this work.

The theory of hydraulic fracturing, indicates that to initialise a fracture fluid injection pressure (pore pressure) needs to be higher than the system compressive stress [16] plus the material strength. In the limit of an homogenous stress distribution, the material is expected to break at an unique pressure value. Figure 4 (b) shows that, as known, in these systems stress is distributed over a large range of values. This means that a fluid pressure below the mean value of the system stress will still be able to mobilise a certain fraction of the particles. It is, therefore, possible to explain the different fracture growth rates (as functions of fluid pressure), or slopes, of the curves in Fig 3 (a) through the standard deviation of the system stress distribution. For a narrow stress distribution a small change in the inlet pressure will allow the mobilisation of a greater fraction of the particles in the system, producing higher slopes for the fracture area vs. inlet pressure curve. A wider stress distribution will, at the same change in the pressure inlet, allow a smaller fraction of the particles to mobilise, this results in the fracture area as a function of inlet pressure "growing" less (smaller slope).

Figure 3 (b) shows the same fracture areas displayed in Fig. 3 (a) but as a function of $\left(p_{i}-p_{c}\right) / \sigma^{-}$, where

$$
p_{c}=C \mu F_{v}+<\operatorname{tr}\left(S^{-}\right)>
$$

It is possible to appreciate the good agreement that all the curves present. The less robust collapse for the lowest value of the lateral force used (red squares in Fig. 3 (b)) is due to the compressive and the cohesive stress being very alike. In this particular system the role of the (otherwise weak) cohesive interaction should be accounted for more carefully.

The differences observed on the fracture growth are also reflected in the fracture geometries. We can characterise this difference in fracture geometry by calculating the radius of gyration $G_{r}^{2}=\left(\sum r_{i}^{2}\right) a r e a^{-1}$ of the fracture patterns presented in Fig 2, which shows that for wider stress distributions (higher lateral forces) the same fracture areas are distributed in a more "compact" way (i.e. more symmetrically distributed around the inlet) than for narrow distributions. This is presented in Fig. 5.

A slow fracture development, where some particles are mobilised with lower fluid pressures, gives to the system the "ability" of relax under the new stress condition, creating "smoother" (less branched) fractures, extending 


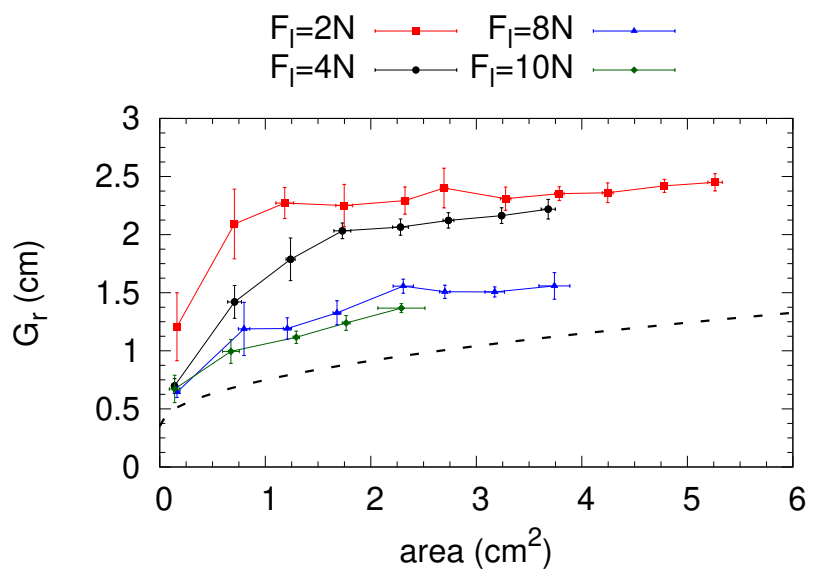

FIG. 5. Radius of gyration $G_{r}$ as a function of the fracture area. Dashed line shows the radius of an area $A$ distributed in a circular shape.

the "symmetric" behaviour regime [1-3] of the fracture development to higher values of fluid pressures.
In summary, we have shown that the characterisation of the stress distribution of an unconsolidated granular packs is fundamental to understand the material mechanical response to stress caused by fluid injection. By distinguishing the nature of the contact forces present in the model we have defined "compressive" and "cohesive" stress tensors. and shown how the development of the hydraulic fracture is closely linked to the standard deviation of the compressive stress distribution. Further work is necessary to assess the relation of the cohesive stress tensor with the material strength and the effect that different consolidation levels have in the fracture behaviour.

\section{ACKNOWLEDGMENTS}

The authors would like to acknowledge the funding and technical support from BP through the BP International Centre for Advanced Materials (BP-ICAM) which made this research possible.
[1] Haiying Huang, Fengshou Zhang, Patrick Callahan, and Joseph Ayoub. Granular fingering in fluid injection into dense granular media in a hele-shaw cell. Physical review letters, 108(25):258001, 2012.

[2] Fengshou Zhang, Branko Damjanac, and Haiying Huang. Coupled discrete element modeling of fluid injection into dense granular media. Journal of Geophysical Research: Solid Earth, 118(6):2703-2722, 2013.

[3] Øistein Johnsen, Renaud Toussaint, Knut Jørgen Måløy, and Eirik Grude Flekkøy. Pattern formation during air injection into granular materials confined in a circular hele-shaw cell. Physical Review E, 74(1):011301, 2006.

[4] D. M Mueth, H. M Jaeger, and S. R Nagel. Force distribution in a granular medium. Phys. Rev. E., 57(3):3164, 1998.

[5] JF Peters, M Muthuswamy, J Wibowo, and A Tordesillas. Characterization of force chains in granular material. Phys. Rev. E., 72(4):041307, 2005.

[6] L Kondic, A Goullet, CS O'Hern, M Kramar, K Mischaikow, and RP Behringer. Topology of force networks in compressed granular media. EPL (Europhys. Lett.), 97(5):54001, 2012.

[7] P. A. Gago, A. Q. Raeini, and P. King. A spatially resolved fluid-solid interaction model for dense granular packs/soft-sand., May 2019.

[8] Christoph Kloss, Christoph Goniva, Alice Hager, Stefan Amberger, and Stefan Pirker. Models, algorithms and validation for opensource dem and $\mathrm{cfd}-\mathrm{dem}$. Progress in
Computational Fluid Dynamics, an International Journal, 12(2-3):140-152, 2012.

[9] Christoph Goniva, Christoph Kloss, Niels G Deen, Johannes AM Kuipers, and Stefan Pirker. Influence of rolling friction on single spout fluidized bed simulation. Particuology, 10(5):582-591, 2012.

[10] Alice Hager, Christoph Kloss, Stefan Pirker, and Christoph Goniva. Parallel open source cfd-dem for resolved particle-fluid interaction. In Proceedings of 9th International Conference on Computational Fluid Dynamics in Minerals and Process Industries, pages 1-6, 2012.

[11] Nikolai V Brilliantov, Frank Spahn, Jan-Martin Hertzsch, and Thorsten Pöschel. Model for collisions in granular gases. Physical review E, 53(5):5382, 1996.

[12] John David Anderson and J Wendt. Computational fluid dynamics, volume 206. Springer, 1995.

[13] Kenneth Langstreth Johnson, Kevin Kendall, and AD Roberts. Surface energy and the contact of elastic solids. Proc. R. Soc. Lond. A, 324(1558):301-313, 1971.

[14] KL Johnson. Continuum mechanics modeling of adhesion and friction. Langmuir, 12(19):4510-4513, 1996.

[15] Paula A Gago, Kuba Wieladek, and Peter King. Fluidinduced fracture into weakly consolidated sand: Impact of confining stress on initialization pressure. Physical Review E, 101(1):012907, 2020.

[16] Fjar Erling, Holt R.M, Horsrud P, Raaen A.M, and Risnes R. Mechanics of hydraulic fracturing. In Petroleum Related Rock Mechanics, pages 1-2. Elsevier, 2nd edition edition, 2008. 